\title{
Optimal tuning pid controller of unstable fractional order system by desired transient characteristics using RIM
}

\author{
Phu Tran Tin', Le Anh Vu², Minh Tran³, Nguyen Quang Dung ${ }^{4}$, Tran Thanh Trang 5 \\ ${ }^{1}$ Faculty of Electronics Technology, Industrial University of Ho Chi Minh City, Vietnam \\ ${ }^{2,3}$ Optoelectronics Research Group, Faculty of Electrical and Electronics Engineering, \\ Ton Duc Thang University, Vietnam \\ ${ }^{4}$ Faculty of Electrical and Electronics Engineering, Ton Duc Thang University, Vietnam \\ ${ }^{5}$ Faculty of Electrical and Electronics Engineering, Ho Chi Minh City University of Food Industry, Vietnam
}

\begin{tabular}{l} 
Article Info \\
\hline Article history: \\
Received Nov 20, 2018 \\
Revised Jan 21, 2019 \\
Accepted Feb 27, 2019 \\
\hline
\end{tabular}

\section{Keywords:}

A distributed parameter system Approximation

Pid

Real interpolation method

\begin{abstract}
In this paper, we propose the method of tuning a conventional PID controller for unstable transient characteristics. The results show that: 1) This is the novel practical method based on the desired settling time and overshoot percentage; 2) The results are close to the desired parameters; 3 ) The novel method can tune an unstable fractional order system by real interpolation method (RIM); 4) The novel method is simplicity and computer efficiency; 5) The novel method can find an optimal solution for tuning task in both academic and industrial purposes.
\end{abstract}

\section{Corresponding Author:}

Le Anh Vu,

Optoelectronics Research Group,

Faculty of Electrical and Electronics Engineering,

Ton Duc Thang University,

Ho Chi Minh City, Vietnam.

Email: leanhvu@tdtu.edu.vn

\section{INTRODUCTION}

Nowadays, PID controllers have received considerable attention in the last years both from an academic and industrial point of view [1-5]. In fact, in principle, they provide more flexibility in the controller design, concerning the standard PID controllers, because they have five parameters to select. However, this also implies that the tuning of the controller can be much more complicated. They have been successfully applied in practical applications such as motion control of manipulators and chaos control of electrical circuits. In these applications, it has been verified that PID controllers can improve the performance of traditional control system adopting integer order PID controllers. The most important advantage of the PID controllers is that they can afford more extensive possibilities offered by their additional fractional order dynamics [4-7]. However, this also indicates that the tuning strategies of PID controllers are much more complicated. In the researches on the PID controllers, tuning of controller parameters has become a significant issue.

In general, the tuning methods for FID controllers are classified into analytical, numerical, and rulebased ones. In [6-7] the controller parameters have been analytically derived by solving nonlinear equations fulfilling the gain/phase crossover frequency and phase/gain margin specifications. The robustness to loop gain variations specification proposed in [8] has also been widely used to design FID and proportionalintegral (PI) controllers. The merits of the analytical method are obvious; however, it is available only when 
the equations are few in number and simple. Therefore, it is very difficult to obtain a complete FID controller for the MB system by solving five complicated nonlinear equations. As for the rule-based method, it can easily calculate the controller parameters based on empirical tuning rules, which can be observed in [9-12]. In this paper, we propose and investigate optimal tuning PID controller of unstable fractional order system by desired transient characteristics using real interpolation method (RIM). The main advantages of this method are drawn as the followings:

a) This is the novel practical method based on the desired settling time and overshoot percentage.

b) The results are close to the desired parameters.

c) The novel method can tune an unstable fractional order system by real interpolation method (RIM).

d) The novel method is simplicity and computer efficiency.

e) The novel method can find an optimal solution for tuning task in both academic and industrial purposes.

The paper is organised as follows. In Section 2 the problem is formulated. The tuning rules are described in Section 3. Simulation results are presented in Section 4, where a comparison with other tuning rules is performed. Conclusions are drawn in Section 5.

\section{RESEARCH METHOD}

\subsection{Real Interpolation Method (RIM)}

The real interpolation method is one of the methods, which works on mathematical descriptions of the imaginary area. The method is based on real integral transform as follow [13]:

$$
F(\delta)=\int_{0}^{\infty} f(t) e^{-\delta t} d t, \delta \in[C, \infty), C \geq 0
$$

which assigns to the original $f(t)$ the image $F(\delta)$ as a function of the real variable $\delta$. Formula of direct transform (12) can be considered as a special case of the direct Laplace transform by replacing the complex variable $p=\delta+j \omega$ for real $\delta$ variable. Another step towards the development of the instrumentation method is the transition from continuous functions $F(\delta)$ to their discrete form, using the computing resources and numerical methods. For these purposes, real interpolation method is represented by numerical characteristics $\left\{F\left(\delta_{i}\right)\right\}_{\eta}$. They are obtained as a set of values of the function $F(\delta)$ in the nodes $i=\overline{1, \eta}$, where $\eta$ is the number of elements numerical characteristics, called its dimension.

Selecting of interpolations $\delta_{i}$ is a primary step in the transition to a discrete form, which has a significant impact on the numerical computing and accuracy of problem solutions. Distribution of nodes in the simplest variant is uniform. Another important advantage of real interpolation method is cross-conversion properties [11]. It dues to the fact that the behavior of the function $F(\delta)$ for large values of the argument $\delta$ is determined mainly by the behavior of the original $f(t)$ for small values of the variable $t$. In the opposite case, the result is the same: the behavior of the function $F(\delta)$ for small values of the argument $\delta$ is determined mainly by the behavior of the original $f(t)$ for large values of the variable $t$ [13].

When considering the original $f(t)$ of dynamic characteristics of dynamic systems, formula (1) leads to an operator model, which under certain conditions can be considered as special cases of the models based on the Laplace transform. Thus, in (1) replacing of the function $f(t)$ by $k(t)$ - the impulse transient function of the dynamic system, we obtain its transfer function. From here we can find the elements of a discrete model of the system, and its transfer function by performing the discretization procedures for nodes $\delta_{i}, i \in \overline{1, \eta}$ :

$$
W\left(\delta_{i}\right)=\int_{0}^{\infty} k(t) e^{-\delta_{i} t} d t, i \in \overline{1, \eta}
$$

Function $W(\delta)$ is a real transfer function of control automatic systems, having an impulse transient response $k(t)$. Function $W(\delta)$ could be received based on determination of transfer function such as a relationship of the imaginary of output $Y(\delta)$ and $X(\delta)$ input signals

$$
W(\delta)=Y(\delta) / X(\delta)
$$

In which the imaginary of the output signal and the input signal is calculated from the original functions of the input $x(t)$ and output $y(t)$ signals: 


$$
\begin{aligned}
& Y(\delta)=\int_{0}^{\infty} y(t) e^{-\delta t} d t \\
& X(\delta)=\int_{0}^{\infty} x(t) e^{-\delta t} d t
\end{aligned}
$$

where $x(t)$ input signal, and $y(t)$ output signal of the system.

The input-output relationship of the system has a form like below:

$$
Y(\delta)=W(\delta) X(\delta)
$$

\subsection{Problem formulation}

In this section, the control system with negative unity feedback is drawn in Figure 1.

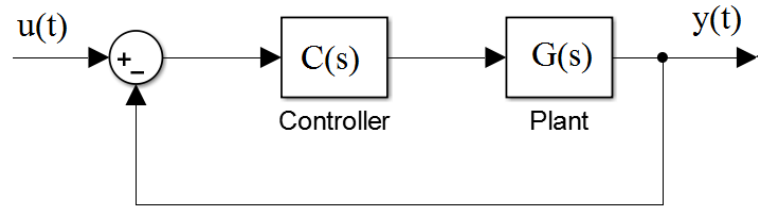

Figure 1. Control system with negative unity feedback

Transfer function of PID controller can be formulated as.

$$
C(\mathrm{~s})=\mathrm{K}_{p}+\frac{\mathrm{K}_{i}}{s}+\mathrm{K}_{d} s
$$

Transfer function of plant (in fractional form) is

$$
\mathrm{G}(\mathrm{s})=\frac{\mathrm{B}_{G}(\mathrm{~s})}{A_{G}(s)}=\frac{b_{n} s^{\beta_{n}}+b_{n-1} s^{\beta_{n-1}}+\cdots+b_{1} s^{\beta_{1}}+b_{0} s^{\beta_{0}}}{a_{m} s^{\alpha_{m}}+a_{m-1} s^{\alpha_{m-1}}+\cdots+a_{1} s^{\alpha_{1}}+a_{0} s^{\alpha_{0}}}
$$

where $m, n \in N$, and $a_{0}, \ldots, a_{m}, b_{0}, \ldots, b_{n}, \alpha_{m}>\alpha_{m-1}>\cdots>\alpha_{1}>\alpha_{0}=0, \beta_{n}>\beta_{n-1}>\cdots>\beta_{1}>\beta_{0}=$ 0 are arbitrary real numbers $a_{m} \neq 0, b_{n} \neq 0$.

Transfer function of PID controller (7) can be rewritten in the rational form as the following

$$
C(\mathrm{~s})=\frac{B_{C}(s)}{A_{C}(s)}=\frac{\mathrm{K}_{d} s^{2}+\mathrm{K}_{p} s+\mathrm{K}_{i}}{s}
$$

Characteristic equation is

$$
\mathrm{W}(s)=1+C(\mathrm{~s}) \mathrm{G}(\mathrm{s})
$$

Characteristic polynomial is

$$
\mathrm{P}(s)=A_{C}(s) A_{G}(s)+B_{C}(s) \mathrm{B}_{G}(s)
$$

A gain-phase margin tester (GPMT) can be thought of as a "virtual compensator", provides information for plotting the boundaries of constant gain margin and phase margin in a parameter plane. The frequency independent GPMT is given in the form [15]:

$$
G_{t}(\mathrm{M}, \phi)=\mathrm{Me}^{-j \phi}
$$


For a given IOPID controller parameters $\mathrm{K}_{p}, \mathrm{~K}_{i}, \mathrm{~K}_{d}$ the closed-loop system is said to be boundedinput bounded-output (BIBO) stable if the quasi-polynomial $\mathrm{P}\left(\mathrm{s}, \mathrm{K}_{p}, \mathrm{~K}_{i}, \mathrm{~K}_{d}\right)$ has no roots in the closed righthalf of the s-plane (RHP). The stability domain $\mathrm{S}$ in the parameter space $\mathrm{P}$ with $\mathrm{K}_{p}, \mathrm{~K}_{i}, \mathrm{~K}_{d}$ being coordinates is the region that for $\mathrm{K}_{p}, \mathrm{~K}_{i}, \mathrm{~K}_{d} \in S$ the roots of quasi-polynomial $\mathrm{P}\left(\mathrm{s}, \mathrm{K}_{p}, \mathrm{~K}_{i}, \mathrm{~K}_{d}\right)$ all lie in open left-half of the s-plane (LHP). The boundaries of the stability domain $\mathrm{S}$ which are described by real root boundary (RRB), infinite root boundary (IRB) and complex root boundary (CRB) can be determined by the Ddecomposition method [14]. These boundaries are defined by the equations $\mathrm{P}(0, \mathrm{~K})=0, \mathrm{P}(\infty, \mathrm{K})=0$ and $\mathrm{P}( \pm \mathrm{j} \omega, \mathrm{K})=0$ for $\omega \in(0, \infty)$, respectively, where $\mathrm{P}(\mathrm{s}, \mathrm{K})$ is the characteristic function of the closed-loop system and $\mathrm{K}$ the vector of controller parameters.

a) Determining RRB

In applying the descriptions of stability boundaries of the stability domain S to the FOCE in (6), the RRB turns out to be simply a straight line given by

$$
\mathrm{P}\left(0, \mathrm{~K}_{p}, \mathrm{~K}_{i}, \mathrm{~K}_{d}\right)=0 \Rightarrow K_{i}=0,
$$

for $s^{\beta_{0}}=1$ in the transfer function of the plant in (8).

b) Determining IRB

There is more theoretical difficulties for the calculating of the IRB due to fractional component. FOCE possesses an infinite number of roots, which cannot be calculated analytically in the general case. However, the asymptotic location of roots far from the origin is well known [21], [22], which may lead to IRB. The objective of this section is to determine the stabilizing region in ( $\mathrm{Kp}, \mathrm{Ki}$ ) plane with given $\mathrm{Kd}$, and values for which the following complex polynomial is

$$
\mathrm{D}(s)=s L(s) A_{G}(s)+\left(\mathrm{K}_{d} s^{2}+\mathrm{K}_{p} s+\mathrm{K}_{i}\right) \mathrm{M}(\mathrm{s}) \mathrm{B}_{G}(s)
$$

where $\mathrm{L}(\mathrm{s})$ and $\mathrm{M}(\mathrm{s})$ are given complex fractional order polynomials. When $\mathrm{L}(\mathrm{s})=\mathrm{M}(\mathrm{s})=1$, the stabilization of (14) reduces to the standard IOPID stabilization.

$$
\mathrm{D}(\infty)=0 \text {. Suppose when } s \rightarrow \infty,\left[L(s) A_{G}(s)\right] /\left[M(s) B_{G}(s)\right] \cdot c s^{\tau} \text { where } \mathrm{t} \text { and } \mathrm{c} \text { are real and complex }
$$
numbers respectively. We have:

(a) If $\tau \neq 1$, then the boundary does not exist.

(b) If $\tau=1$ and $\mathrm{c}$ is not real, then the boundary does not exist.

(c) If $\tau=1$ and $\mathrm{c}$ is real, then

$$
\begin{aligned}
& \lim _{s \rightarrow \infty} \frac{D(s)}{s L(s) A_{G}(s)}=\lim _{s \rightarrow \infty} 1+\frac{N(s)\left(K_{p} s+K_{i}+K_{d} s^{2}\right)}{s A_{G}(s)}=1+K_{d} c=0 \\
& K_{d}=-\frac{1}{c}
\end{aligned}
$$

c) Determining CRB

To construct the CRB, we substitute $s=j \omega$ into (11) to obtain,

$$
\mathrm{P}(\mathrm{j} \omega)=A_{C}(\mathrm{j} \omega) A_{G}(\mathrm{j} \omega)+B_{C}(\mathrm{j} \omega) \mathrm{B}_{G}(\mathrm{j} \omega)=0
$$

Using D-decomposition to find stability [21], to construct the CRB, we substitute ${ }^{s=j \omega}$ into (11) to obtain

$$
\mathrm{P}(\mathrm{j} \omega)=A_{C}(\mathrm{j} \omega) A_{G}(\mathrm{j} \omega)+B_{C}(\mathrm{j} \omega) \mathrm{B}_{G}(\mathrm{j} \omega)=0
$$

Using D-decomposition to find stability [ [1] Reference -A graphical tuning]

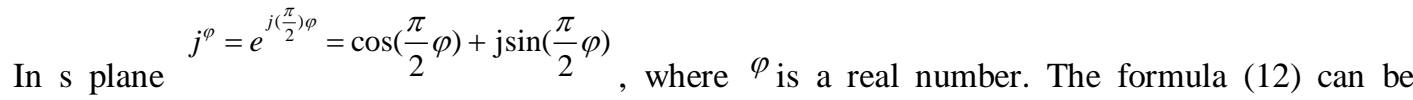
expressed into a formula with the separate real and imaginary components 


$$
\mathrm{P}(\mathrm{j} \omega)=\left[\left(\mathrm{K}_{i}-\mathrm{K}_{d} \omega^{2}\right) \mathrm{R}_{B G}(\omega)-\omega \mathrm{I}_{A G}(\omega)\right]+j \omega\left[\mathrm{K}_{p} \mathrm{R}_{B G}(\omega)+\mathrm{R}_{A G}(\omega)\right]=0
$$

where $\mathrm{R}_{B G}, \mathrm{R}_{A G}, \mathrm{I}_{A G}$ are the real component of $\mathrm{B}_{G}(\mathrm{j} \omega)$, the real and imaginary components of $A_{G}(\mathrm{j} \omega)$, respectively. Finally, by setting the real and imaginary parts equalized zero the formula (15) leads to the detail form:

$$
\left\{\begin{array}{l}
\mathrm{R}_{A G}(\omega)+\mathrm{K}_{p} \mathrm{R}_{B G}(\omega)=0 \\
\left(\mathrm{~K}_{i}-\mathrm{K}_{d} \omega^{2}\right) \mathrm{R}_{B G}(\omega)-\omega \mathrm{I}_{A G}(\omega)=0
\end{array}\right.
$$

The CRB is determined by formula (17).

\subsection{Algorithm}

\subsubsection{Design of the desired transient characteristics}

Performance qualities of the control system can be evaluated by settling time, overshot percentage, damping ratio, natural frequency, rise time. There are some methods to design the desired transfer characteristics [18-20]. In this paper, the desired transient characteristics could be used a second order system. A second order system can be described by the following form without zeros

$$
H(s)=\frac{\omega_{n}^{2}}{s^{2}+2 \zeta \omega_{n} s+\omega_{n}^{2}}
$$

With zeros,

$$
H(s)=\frac{s \omega_{n} /(\alpha \zeta)+\omega_{n}^{2}}{s^{2}+2 \zeta \omega_{n} s+\omega_{n}^{2}}
$$

Where ${ }^{\omega_{n}}$ natural frequency, $\zeta$ damping ratio, $\alpha$ coefficient related to overshoot percentage. And there is a relationship between settling time and natural frequency and damping ratio

$$
t_{s e t} \approx \frac{4}{\omega_{n} \zeta}
$$

Another option is that we can use the mat lab or another software program to design a desired transient characteristics.

\subsubsection{Real interpolation method}

The desired transient characteristics could be given by massive data, table and transient function. Let see the transient characteristics given by form: function $h_{D}(t)=f(t)$ or massive $h_{D i}=f\left(t_{i}\right)$

From Figure 1, the equivalent transfer function of the feedback system has a form

$$
W(s)=\frac{C(s) G(s)}{1+C(s) G(s)}
$$

Or it could be rewritten by input and output transfer function

$$
\frac{Y(s)}{X(s)}=\frac{C(s) G(s)}{1+C(s) G(s)}
$$

If the input of the tuning system is a step function, when $X(s)$ is determined

$$
X(s)=1 / \mathrm{s}
$$


To determine coefficient $\mathrm{Kp}, \mathrm{Ki}, \mathrm{Kd}$ in PID controller for fractional order transfer function using the real interpolation method. By substituting $s=\delta$ formula (22) can be rewritten in the real transfer form.

$$
\frac{Y(\delta)}{X(\delta)}=\frac{C(\delta) G(\delta)}{1+C(\delta) G(\delta)}
$$

From (24) we obtain the formula determined PID controller.

$$
C(\delta)=\frac{1}{G(\delta)[\mathrm{X}(\delta) / \mathrm{Y}(\delta)-1]}
$$

where $G(\delta) \mathrm{X}(\delta) \mathrm{Y}(\delta)$ real transfer function of the plant, the imaginary of output and input signals respectively.

The functions $\mathrm{X}(\delta), Y(\delta)$ could be determined by formula (4) and (5) in the real form or by following formula. If the transient characteristics are given by a massive the functions $\mathrm{X}(\delta), Y(\delta)$ can be obtained by the following formula:

$$
\begin{aligned}
& X\left(\delta_{i}\right)=\sum_{i=1}^{N} x\left(t_{i}\right) e^{-\delta_{i} t_{i}} \Delta t \\
& Y\left(\delta_{i}\right)=\sum_{i=1}^{N} h\left(t_{i}\right) e^{-\delta_{i} t_{i}} \Delta t
\end{aligned}
$$

where $x\left(t_{i}\right)$ and $h\left(t_{i}\right)$ are the input value and the output value at time ${ }^{t_{i}}$, respectively; $\Delta t$-the sample time and $\mathrm{N}$ - the number of samplings.

If the input of the tuning system is a simple step function, when $X(\delta)$ is determined in real form.

$$
X(\delta)=1 / \delta
$$

The number of the unknown coefficients of the PID controller $\left(K_{p}, K_{i}, K_{d}\right)$ is three; it means that the dimension of the elements numerical characteristics is three $(\eta=3)$.Selecting value of nodes is a primary step. It effects on the accuracy of the tuning task. The meaningful region is from 0 to ${ }^{t}{ }^{s e t}{ }^{(s)}$. It means that the maximum value of first node ${ }^{\delta_{1}}$ can be defined by the following condition: the value of the integral in (4) by the settling time ty reduces to a negligible value $\Delta=0.001 \div 0.05$, which satisfy the condition $h\left(t_{\text {set }}\right) e^{\delta_{1} t_{\text {set }}} \leq \Delta$. Hence calculated expression for the node ${ }^{\delta_{1}}$ can be shown below.

$$
\delta_{1}=-\frac{\ln \left(\Delta / h\left(t_{\text {set }}\right)\right)}{t_{\text {set }}}
$$

where $\Delta$-calculation error, ${ }^{h\left(t_{\text {set }}\right)}$ - settling value, ${ }^{t_{\text {set }}}$ - settling time.

Other node in the simplest case, can be determined by the nominal distribution.

$$
\delta_{i}=i \delta_{1}, i=\overline{2, \eta}
$$

In case, the result according to the maximum value of the first node do not meet the desired output performances, the value of the first node should be decreased. In this paper, by varying the value of the first node from the maximum, determined by (29) approach to 0 , we carry out the optimization of the range of nodes for the output requirements of the desired system.

The numerical characteristics of the PID controller's real transfer function. 


$$
\begin{aligned}
& C\left(\delta_{i}\right)=\mathrm{K}_{p}+\frac{\mathrm{K}_{i}}{\delta_{i}}+\mathrm{K}_{d} \delta_{i}, \quad i=\overline{1, \eta} \\
& \mathrm{F}\left(\delta_{i}\right)=\frac{1}{G\left(\delta_{i}\right)\left[\mathrm{X}\left(\delta_{i}\right) / \mathrm{Y}\left(\delta_{i}\right)-1\right]}, \quad i=\overline{1, \eta}
\end{aligned}
$$

We have a system of equations with unknown coefficient $K_{p}, K_{i}, K_{d}$

$$
C=\left[\begin{array}{ccc}
1 & \frac{1}{\delta_{1}} & \delta_{1} \\
1 & \frac{1}{\delta_{2}} & \delta_{2} \\
1 & \frac{1}{\delta_{3}} & \delta_{3}
\end{array}\right], \quad K=\left[\begin{array}{l}
K_{p} \\
K_{i} \\
K_{d}
\end{array}\right], \quad F=\left[\begin{array}{l}
\mathrm{F}\left(\delta_{1}\right) \\
\mathrm{F}\left(\delta_{2}\right) \\
\mathrm{F}\left(\delta_{3}\right)
\end{array}\right]
$$

The unknown coefficients $K_{p}, K_{i}, K_{d}$ can be obtained in matrix form

$$
K=C^{-1} F
$$

Algorithm:

a) Determination of stability region (13), (16) and (17).

b) Design a desired transient characteristic (18) or (19)

c) Calculation value of nodes (29) and numerical characteristics (22) or (28), (27), (31) and (32).

d) Solving the equation to find the parameters of PID controller (33) and (34).

e) Estimation of the tuned PID controller in the time and frequency domains.

f) Investigation of the optimal range of the values of node by varying the value of the nodes.

\section{NUMERICAL RESULTS AND DISCUSSION}

Given fractional order transfer function of a unstable bearing system [zhong16],

$$
\mathrm{G}(\mathrm{s})=\frac{6438 .}{s^{2.76}+330.04 s^{1.81}-84268.85 s^{0.82}-15869154.14}
$$

Investigation of STABILITY REGION of the unstable fractional order transfer function of the bearing system (11).

a) Determine RRB

It is important to determine the stability region of the PID controller, tuned for the system (35). The RRB can be determined by (13) as the bellow

$$
K_{i}=0
$$

b) Determine IRB

The IRB can be determined according to (16)

$$
\left.\lim _{s \rightarrow \infty} \frac{\delta(s)}{s D(s)}=\lim _{s \rightarrow \infty} 1+\frac{643.8\left(K_{p} s+K_{i}+K_{d} s^{2}\right)}{s()}\right)=1
$$

Not exist the IRB.

c) Determine CRB

The CRB can be determined by three conditions (18), (19) and (20). The results are shown in the following figures. The following figure shows the stability region of the transfer function (11). The CRB in $(\mathrm{Kp}, \mathrm{Ki})$ plane when $\mathrm{Kd}=10$ is shown in the Figure 2 . The cross-line area is a feasible region for the coefficients of the PID controller. 


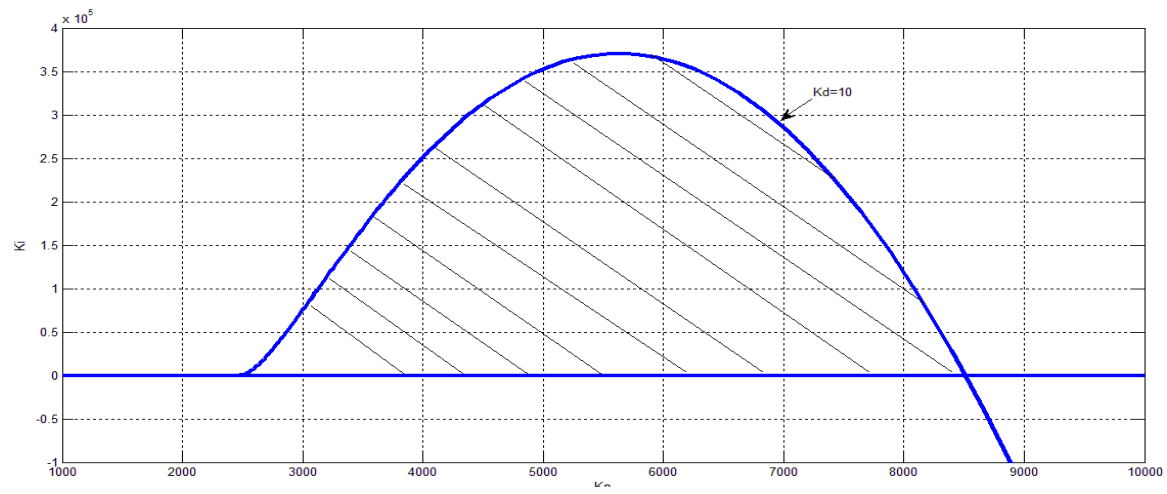

Figure 2. The feasible region of coefficients $\mathrm{Kp}, \mathrm{Ki}, \mathrm{Kd}$

The cross-line region demonstrates stability region of $\mathrm{Kp}, \mathrm{Ki}$ where $\mathrm{Kd}=10$. Oviously, the stability region of PID controller's coefficients lie in the first quarter. In the Figure 3 there are shown more feasible area with five values of $\mathrm{Kd}$.

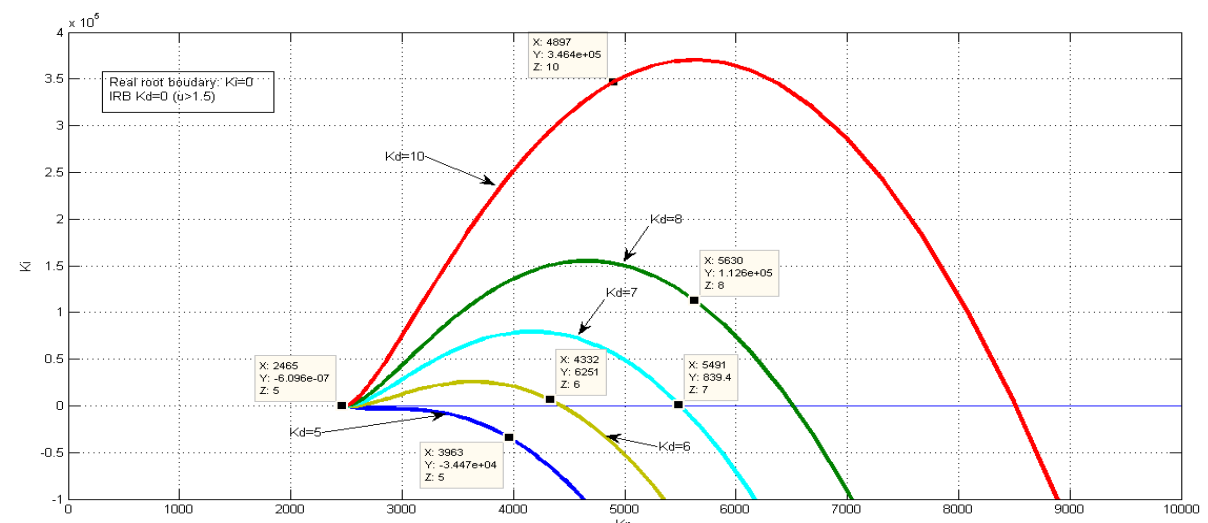

Figure 3. Stability region of PID controller depending on its coefficients

All boundaries (RRB, IRB and CRB) are shown in following figure. Kp, Ki, Kd stability region The results simply lead to the conclusion

$$
\left\{\begin{array}{l}
K_{p}>=2465 \\
K_{i}>0
\end{array}\right.
$$

Three parameters of $K_{p}, K_{i}, K_{d}$ are located in the first quarter of coordinate axis system. STUDY CASE 1

Design of the desired transient characteristics.

Using (22) and (23) with the desired transient time $T_{\text {set }}=0.03 \mathrm{~s}$ and overshoot $P O T=50 \%$ we formed a transfer function with the step response shown in the Figure 4. According to Figure 4, the transient time of the desired system accounts for $T_{\text {set }}=0.0366 \mathrm{~s}$ and the overshoot is given a desired value at $P O T=50.1 \%$. 


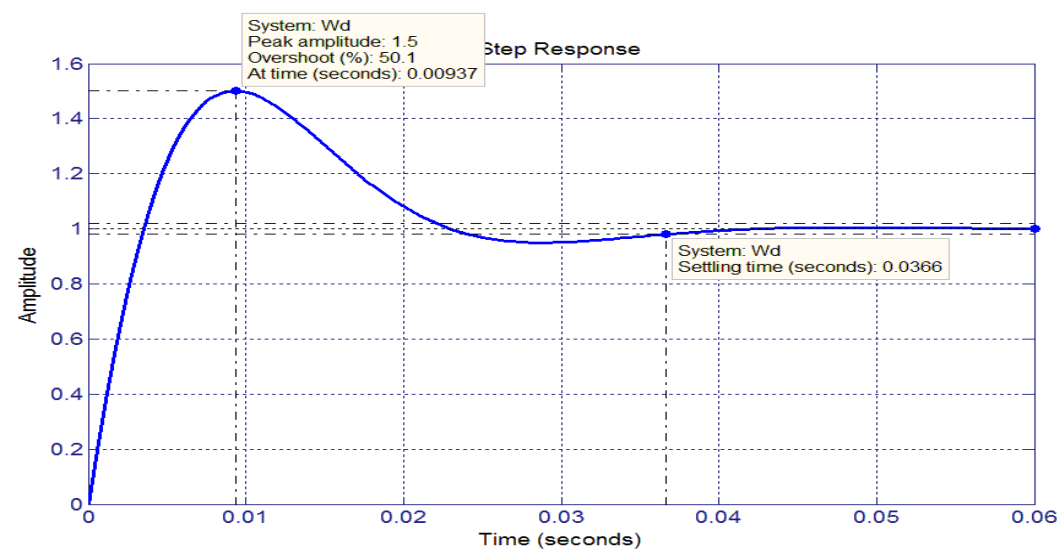

Figure 4. The desired transient characteristics with overshoot percentage $50.1 \%$ and settling time $0.0366 \mathrm{~s}$

Obviously, the number of the numerical characteristics is three $(\eta=3$ ) because of three unknown coefficients of the PID controller. Calculation of the nodes of numerical characteristics:

Using the formula (30) with the input parameters $t_{\text {set }}=0.036 \mathrm{~s}, \Delta t=0.001 \mathrm{~s}, \Delta=0.001, h_{\text {set }}=1$ the first node can be determined $\delta_{1}=188.736$. Other nodes are determined by the condition of uniform distribution: $\delta_{i}=i \delta_{1}, i=\overline{2, \eta}$. Finally, the value of nodes can show the following massive $\delta_{i}=[188.736377 .473$ 566.209]

Based on the finding nodes the value of massive $\mathrm{F}$ can calculated and make up $F_{i}=[-25230402.2196006]$, and $\mathrm{C}$ massive accounts for

$$
C=\left[\begin{array}{ccc}
1 & 5.298 \cdot 10^{-3} & 188.736 \\
1 & 2.649 \cdot 10^{-3} & 377.473 \\
1 & 1.7660 \cdot 10^{-3} & 566.209
\end{array}\right] \text { and the coefficients of the PID controller are } K=\left[\begin{array}{l}
39271.902 \\
-1.134 \cdot 10^{7} \\
-23.375
\end{array}\right] .
$$

According to the feasible area (39) of PID controller the values of the tuned parameters of the controller are not satisfied. When $\mathrm{d} 1=\{2.5,5,5,7.5,10$ the results are shown in the Figure 5 .

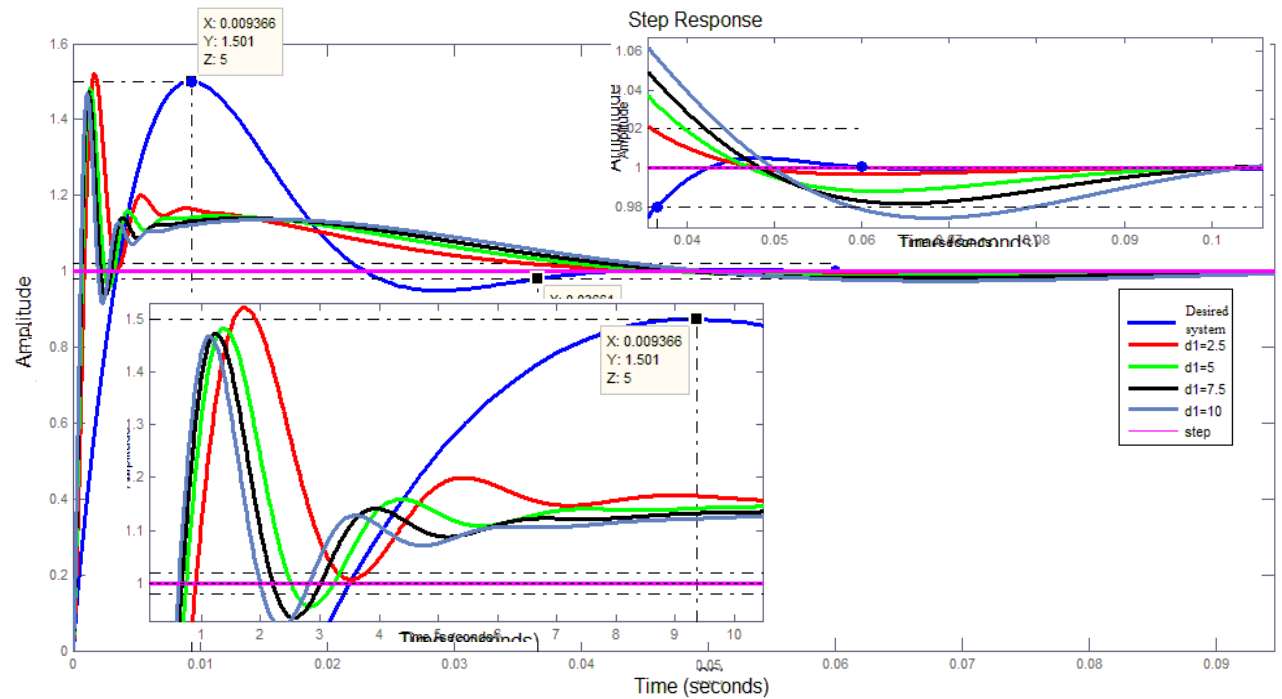

Figure 5. Time response of the tuning systems 
In terms of the percentage overshot, the tuning systems are close to the desired system. With $\delta 1=2.5$ the percentage overshot of the tuning system is slightly higher than the expected value. With other values of $\delta 1$ the figure is lower than that of the expected value with the higher value of the first node. According to the settling time, the values of the tuning systems are greater than that of the desired system.

On the other hand, the shape of the time response figures do not following the desired transient response. The bode graph show in the following figure. It is clear that the bode graphs of the tuning systems are close to each other. The magnitude and phase margin of the tuning system are close to each other. The bode diagram of the open loop system is shown in Figure 6.

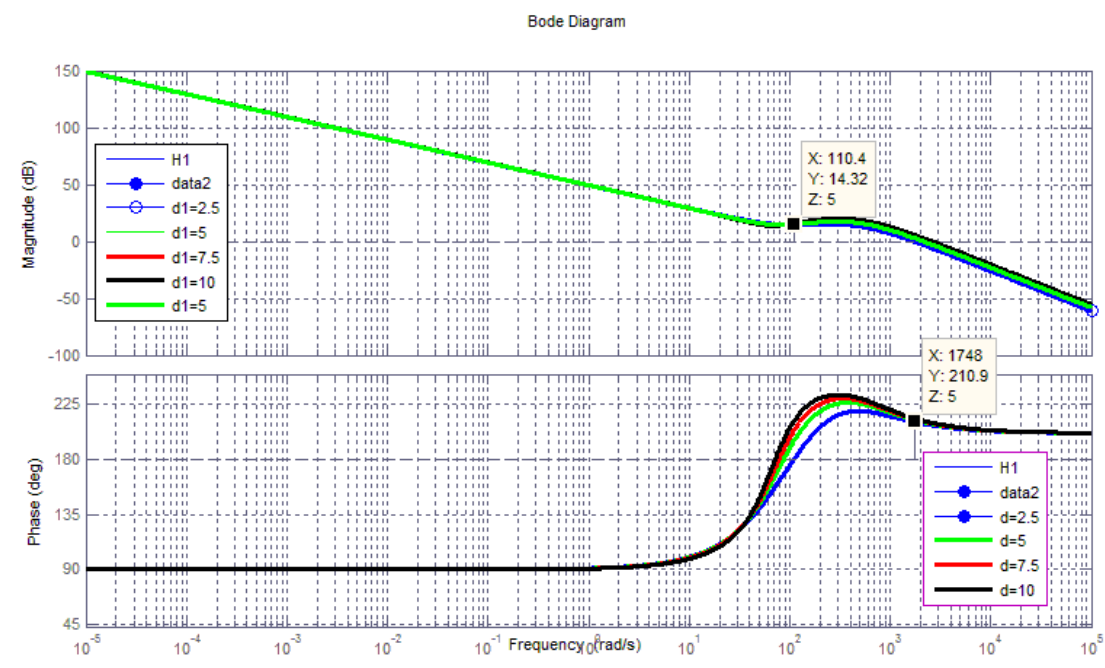

Figure 6. Bode of the open loop system

The detail values of the tuning system are presented in the following table. STUDY CASE 2

We carry out the investigation for the higher value of the desired overshoot percentage and settling time. The desired overshoot percentage and settling time account for $100 \%$ and $0.0429 \mathrm{~s}$ respectively. The desired transient process is shown in the Figure 8. The desired transient characteristics with overshoot percentage $99.1 \%$ and settling time $0.0429 \mathrm{~s}$.

The results of the tuning process will illustrate in the Figure 7 . When $\delta 1=5,10,20,50$ the time responses are shown in the following Figure 7.

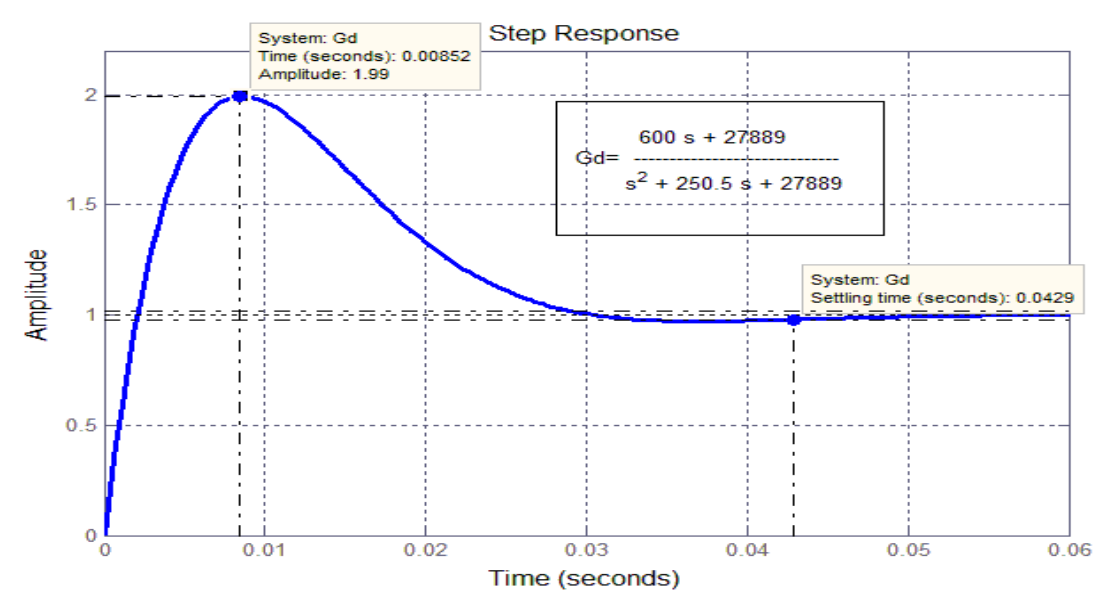

Figure 7. The desired transient characteristics with overshoot percentage $99.1 \%$ and settling time $0.0429 \mathrm{~s}$ 


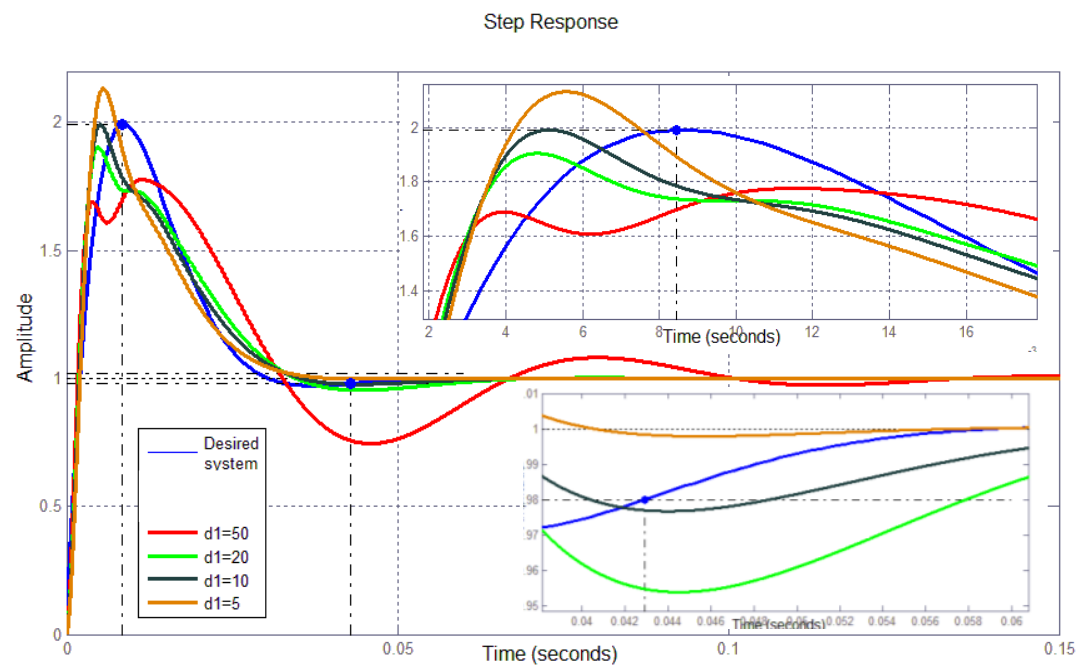

Figure 8. Time response of the tuning systems

The shapes of the transient response of the tuning systems are closer to the desired system than that in the first example. Higher accuracy than that of the first example. Bode graph of the tuning systems is shown in the Figure 9.

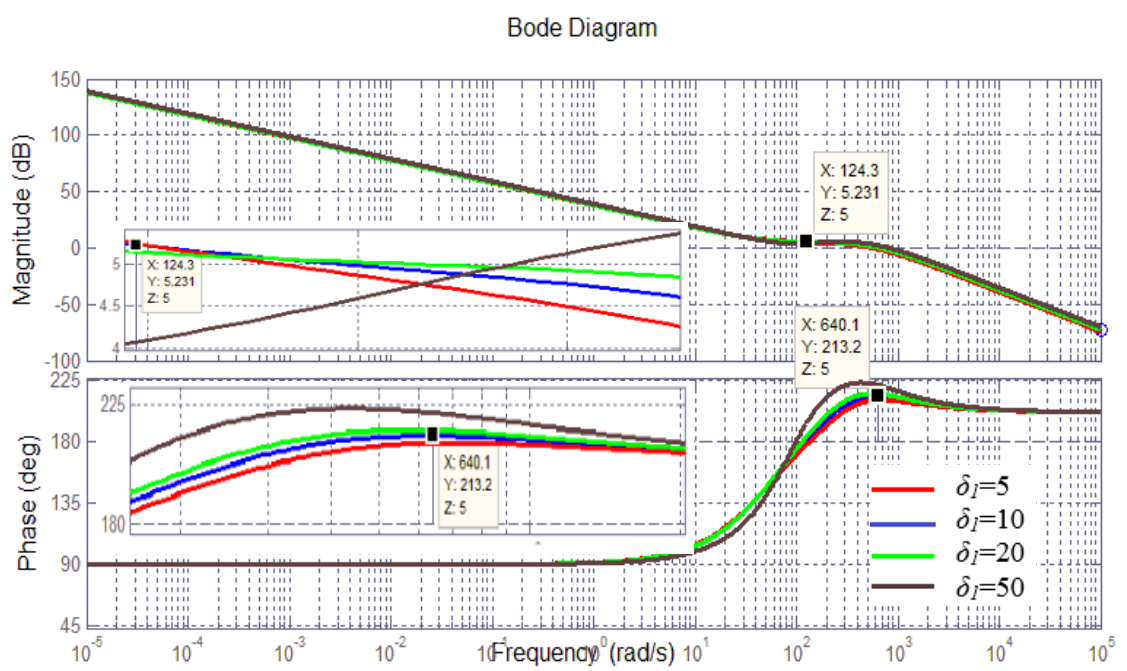

Figure 9. Bode graph of the tuning systems

The magnitude margins show the same value for all tuning system, however, the phase magnitude margins account for higher value with the higher value of the first node.

\section{CONCLUSION}

In this paper, we propose the method of tuning conventional PID controller for unstable transient characteristics. The results show that: 1) This is the novel practical method based on the desired settling time and overshoot percentage; 2) The results are close to the desired parameters; 3) The novel method can tune an unstable fractional order system by real interpolation method (RIM); 4) The novel method is simplicity and computer efficiency; 5) The novel method can find an optimal solution for tuning task in both academic and industrial purposes. 


\section{REFERENCES}

[1] Heydarpoor, Saeed, and Mohammad Tabatabaei. "Velocity Control of a DC Motor Based on Fractional Order PI and IMC-based Fractional Order Controllers." International Journal of Advanced Mechatronic Systems 7, no. 1 (2016): 35. doi:10.1504/ijamechs.2016.079650.

[2] Zheng, Shiqi. "Robust Stability of Fractional Order System with General Interval Uncertainties." Systems \&amp; Control Letters, vol. 99, 2017, pp. 1-8., doi:10.1016/j.sysconle.2016.11.001.

[3] Vinagre, Blas M., et al. "Fractional PID Controllers for Industry Application. A Brief Introduction." Journal of Vibration and Control, vol. 13, no. 9-10, 2007, pp. 1419-1429., doi:10.1177/1077546307077498.

[4] Monje, Concepción A., et al. "Tuning and Auto-Tuning of Fractional Order Controllers for Industry Applications." Control Engineering Practice, vol. 16, no. 7, 2008, pp. 798-812. doi:10.1016/j.conengprac.2007.08.006.

[5] Chen, Yangquan, et al. "Practical Tuning Rule Development for Fractional Order Proportional and Integral Controllers." Journal of Computational and Nonlinear Dynamics, vol. 3, no. 2, 2008, p. 021403., doi:10.1115/1.2833934.

[6] Petráš, Ivo. "Tuning and Implementation Methods for Fractional-order Controllers." Fractional Calculus and Applied Analysis 15, no. 2 (01, 2012). doi:10.2478/s13540-012-0021-4.

[7] Li, Hongsheng, Ying Luo, and Yangquan Chen. "A Fractional Order Proportional and Derivative (FOPD) Motion Controller: Tuning Rule and Experiments." IEEE Transactions on Control Systems Technology 18, no. 2 (03 2010): 516-20. doi:10.1109/tcst.2009.2019120.

[8] Luo, Ying, Chunyang Wang, and Yangquan Chen. "Tuning Fractional Order Proportional Integral Controllers for Fractional Order Systems." 2009 Chinese Control and Decision Conference, 062009. doi:10.1109/ccdc.2009.5195101.

[9] Malek, Hadi, Ying Luo, and Yangquan Chen. "Identification and Tuning Fractional Order Proportional Integral Controllers for Time Delayed Systems with a Fractional Pole." Mechatronics 23, no. 7 (10 2013): 746-54. doi:10.1016/j.mechatronics.2013.02.005.

[10] Valério, Duarte, and José Sá Da Costa. "Tuning of Fractional PID Controllers with Ziegler-Nichols-type Rules." Signal Processing 86, no. 10 (10 2006): 2771-784. doi:10.1016/j.sigpro.2006.02.020.

[11] Bhambhani, Varsha, Yangquan Chen, and Dingyü Xue. "Optimal Fractional Order Proportional Integral Controller for Varying Time-Delay Systems." IFAC Proceedings Volumes 41, no. 2 (2008): 4910-915. doi:10.3182/200807065-kr-1001.00825.

[12] Chen, Yangquan, Tripti Bhaskaran, and Dingyü Xue. "Practical Tuning Rule Development for Fractional Order Proportional and Integral Controllers." Journal of Computational and Nonlinear Dynamics 3, no. 2 (2008): 021403. doi:10.1115/1.2833934.

[13] Goncharov, Nguyen Quang Dung, Than Viet Dung, Identification of object with distributed parameters, Theory and practice in the physical, mathematical and technical sciences-XXIV International Scientific and Practice Conference, London, May, 2012. doi:10.18007/gisap:tsca.v0i8.1421.

[14] Strakos, Premysl, and Jiri Tuma. "Mathematical Modelling and Controller Design of Inverted Pendulum." 2017 18th International Carpathian Control Conference (ICCC), 05 2017. doi:10.1109/carpathiancc.2017.7970431.

[15] Hamamci, S.e. "An Algorithm for Stabilization of Fractional-Order Time Delay Systems Using Fractional-Order PID Controllers." IEEE Transactions on Automatic Control 52, no. 10 (10 2007): 1964-969. doi:10.1109/tac.2007.906243.

[16] Zhong, Jianpeng, and Lichuan Li. "Tuning Fractional-Order <Italic/><Inline-Formula $\rangle$ Tex-Math Notation='LaTeX’ $>\$\{\mathrm{PI}\}^{\wedge}\{\lfloor$ Lambda $\}\{\mathrm{D}\} \wedge\{\mathrm{IMu}\} \$</$ Tex-Math $></$ Inline-Formula $>$ Controllers for a Solid-Core Magnetic Bearing System." IEEE Transactions on Control Systems Technology, vol. 23, no. 4, 2015, pp. 1648-1656., doi:10.1109/tcst.2014.2382642.

[17] Zheng, Shiqi, Xiaoqi Tang, and Bao Song. "A Graphical Tuning Method of Fractional Order Proportional Integral Derivative Controllers for Interval Fractional Order Plant." Journal of Process Control 24, no. 11 (11 2014): 1691-709. doi:10.1016/j.jprocont.2014.08.011.

[18] Rashid, Tarique, Sunil Kumar, Akshay Verma, Prateek Raj Gautam, and Arvind Kumar. "Pm-EEMRP: Postural Movement Based Energy Efficient Multi-hop Routing Protocol for Intra Wireless Body Sensor Network (IntraWBSN)." TELKOMNIKA (Telecommunication, Computing, Electronics and Control) 16, no. 1 (02, 2018): 166. doi:10.12928/telkomnika.v16i1.7318.

[19] A. F. Morabito, "Power Synthesis of Mask-Constrained Shaped Beams Through Maximally-Sparse Planar Arrays," Telkomnika (Telecommunication Computing Electronics and Control), vol. 14, n. 4, pp. 1217-1219, 2016.

[20] Phu Tran Tin, Tran Hoang Quang Minh, Tran Thanh Trang, Nguyen Quang Dung "Using Real Interpolation Method for Adaptive Identification of Nonlinear Inverted Pendulum System". International Journal of Electrical and Computer Engineering (IJECE), Vol. 9, No. 2, April 2019, pp. 1078 1089: DOI: 10.11591/ijece.v9i2. pp.1078-1089. 